\title{
CD5L-induced activation of autophagy is associated with hepatoprotection in ischemic reperfusion injury via the CD36/ATG7 axis
}

\author{
JUNJIAN LI ${ }^{1}$, WEI LIN ${ }^{2,3}$ and LEI ZHUANG ${ }^{1}$ \\ ${ }^{1}$ Department of Hepatobiliary Surgery, The First Affiliated Hospital, Wenzhou Medical University; \\ ${ }^{2}$ Science and Technology Information Center, Wenzhou Medical University Library; ${ }^{3}$ Department of Geriatrics, \\ The First Affiliated Hospital, Wenzhou Medical University, Wenzhou, Zhejiang 325000, P.R. China
}

Received August 14, 2018; Accepted July 24, 2019

DOI: $10.3892 /$ etm.2020.8497

\begin{abstract}
Hepatic ischemia/reperfusion (I/R) injury is a side effect of major liver surgery that is difficult to prevent. I/R injury induces metabolic strain on hepatocytes and limits the tolerable ischemia during liver resection, as well as preservation times during transplantation. Additionally, I/R injury induces apoptosis in hepatocytes. CD5-like (CD5L), an inducer of autophagy, is a soluble scavenger cysteine-rich protein that modulates hepatocyte apoptosis. The aim of the present study was to determine if pharmacologic CD5L was protective against hepatic ischemia-reperfusion injury. Hepatocytes were subjected to I/R culture conditions, and apoptosis and caspase family activity were measured after I/R to model hepatic injury. Treatment with recombinant CD5L significantly suppressed apoptosis and caspase activity through modulating cellular autophagy to maintain activation of the cluster of differentiation 36 (CD36)-dependent autophagy-related 7 (ATG7) signaling pathway. The regulation loop between CD5L and the autophagy signaling pathway was identified to be associated with the inhibition of oxidative stress. Treatment with CD5L significantly inhibited cellular oxidative stress, which was confirmed by silencing the CD36 receptor or the autophagy related protein ATG7 using small interfering RNA, which reversed the antiapoptotic and antioxidative effects of $\mathrm{CD} 5 \mathrm{~L}$ on hepatocytes under I/R conditions. The results of the present study suggested that CD5L-mediated attenuation of hepatic I/R injury occurs through the CD36-dependent ATG7 pathway, accompanied by the inhibition of oxidative stress, which is associated with enhanced autophagy. In conclusion,
\end{abstract}

Correspondence to: Dr Lei Zhuang, Department of Hepatobiliary Surgery, The First Affiliated Hospital, Wenzhou Medical University, 2 FuXue lane, Wenzhou, Zhejiang 325000, P.R. China

E-mail: 543933862@qq.com

Key words: apoptosis, autophagy, CD5L, hepatic ischemia/reperfusion injury, oxidative stress the present study identifies CD5L as a novel therapeutic agent for hepatic I/R injury.

\section{Introduction}

For patients with hepatic malignancy, liver resection may often be the only treatment option (1). Excessive blood loss during surgery is associated with poor postoperative outcomes (2); to avoid this, vascular inflow occlusion (VIO) is often performed during liver transaction (3). Although VIO effectively reduces blood loss, hepatic oxygen supply is interrupted, which results in metabolic disruption that subjects the liver to hepatic ischemia/reperfusion (I/R) injury once oxygen is reintroduced (4).

Apoptosis is a process of programmed cell death that serves an important role in the progression of I/R injury (5). Intrinsic apoptosis, also termed the mitochondrial apoptosis pathway, is induced by intracellular stress, such as oxidative stress, and subsequent activation of the caspase family-mediated apoptotic cascade (6). Apoptotic hepatocytes are observed in liver I/R injury (7). Pharmacological inhibition of hepatocyte apoptosis has been demonstrated to improve I/R injury $(8,9)$. Therefore, targeting apoptosis may be a promising preventive and therapeutic strategy for hepatic I/R injury.

CD5-like (CD5L) protein is a soluble glycoprotein, also known as apoptosis inhibitor of macrophage (AIM) (10). CD5L serves diverse roles in the relationship between lipid homeostasis and the immune response (11). CD5L localizes to cell surface receptor cluster of differentiation 36 (CD36) to promote the transcription of genes involved in the regulation of mitochondrial biogenesis to maintain energy and metabolic homeostasis; thus, CD5L may promote antiapoptotic effects in hepatocellular carcinoma $(12,13)$. Therefore, exogenous CD5L may have beneficial effects in protecting liver from I/R induced injury.

The autophagy salvage pathway is an additional means of energy generation in cells and can be activated by various cellular stressors, including I/R (14). Autophagy is a process of degradation and recycling of large molecules and dysfunctional organelles, protecting cells from apoptosis (15). As an inducer of autophagy, CD5L may serve a functional role in cytoprotection processes in macrophages and hepatocytes $(12,16)$. 
Hepatic I/R injury is a sterile inflammatory response that follows hepatic ischemia and is characterized by overproduction of reactive oxygen species (ROS) followed by hepatocyte apoptosis $(17,18)$. During ischemia, the absence of oxygen leads to an accumulation of ROS and a reduction in antioxidative enzymes (19). A previous study has suggested that CD5L may promote an anti-inflammatory cytokine profile through the modulation of autophagy, leading to an inhibition of ROS generation (20). The present study investigated whether CD5L was able to modulate cellular oxidative stress to alleviate the I/R injury.

The aim of the present study was to determine whether exogenous CD5L would enhance autophagy through the CD36 receptor and decrease I/R-induced oxidative stress, leading to inhibition of hepatocyte apoptosis, thereby attenuating hepatic $\mathrm{I} / \mathrm{R}$ injury.

\section{Materials and methods}

Isolation, culture and treatment of hepatocytes. All animals received humane care according to protocols approved by the institutional care and use committee at the Wenzhou Medical University (Ethics Committee of Wenzhou Medical University, Wenzhou, China; approval no. WMU18825). A total of $60 \mathrm{C} 57 \mathrm{BL} / 6$ mice were used for the present study. The housing conditions for the mice were as follows: Light/dark cycle, $12-\mathrm{h}$; temperature, $21 \pm 2^{\circ} \mathrm{C}$; relative humidity, $30-70 \%$; food and water, freely available to each animal throughout. Mouse hepatocytes were isolated from 3-month-old male C57BL/6 mice ( $n=12 /$ group) using the collagenase perfusion method as previously described (21). Briefly, mice were anesthetized with $3.5 \%$ chloral hydrate $(10 \mu \mathrm{l} / \mathrm{g}$ body weight; $350 \mathrm{mg} / \mathrm{kg}$ ). Following a laparotomy, the vena cava was catheterized. The liver was perfused with pre-warmed EGTA buffer followed by collagenase buffer. The liver was immediately dissected and placed in a $10-\mathrm{cm}$ cell culture dish for mincing. The separated hepatocytes were filtered through a $70-\mu \mathrm{m}$ cell strainer to remove tissue debris. Hepatocytes were washed twice with cold low-glucose $(5.5 \mathrm{mM})$ Dulbecco's modified Eagle's medium (DMEM; Gibco; Thermo Fisher Scientific, Inc.) containing $10 \%$ fetal bovine serum and centrifuged at $50 \mathrm{x}$ g for $5 \mathrm{~min}$ at $4^{\circ} \mathrm{C}$.

For experiments involving hypoxia, the medium was replaced with serum-free DMEM/F12 medium (Gibco; Thermo Fisher Scientific, Inc.) saturated with $95 \% \mathrm{~N}_{2} / 5 \% \mathrm{CO}_{2}$ at $37^{\circ} \mathrm{C}$. The cells were placed in an experimental hypoxia chamber in a saturated atmosphere of $95 \% \mathrm{~N} 2$ and $5 \% \mathrm{CO}_{2}$. To simulate re-oxygenation, hepatocytes were cultured under normal conditions, as previously described (9). Following I/R injury, hepatocytes adhered to the culture dish; $0.25 \%$ trypsin-EDTA at $37^{\circ} \mathrm{C}$ was used to release them from the plate.

For CD5L treatment, cells (I/R model) were cultured with DMEM/F12 containing $1 \mu \mathrm{g} / \mathrm{ml}$ recombinant CD5L and incubated at $37^{\circ} \mathrm{C}$ for $1 \mathrm{~h}$ before cell I/R treatment as previously described (16).

Flow cytometry. Flow cytometry using Annexin V-PI Apoptosis Detection kit (BD Biosciences) was performed to assess apoptosis as described previously (22). Hepatocytes
(I/R model; $1 \times 10^{5}$ cells/well) were stained with Annexin V and propidium iodide according to the manufacturer's protocols. Data were acquired on a FACScan instrument (BD Biosciences) and analyzed using CellQuest software (version 8.0.1; BD Biosciences).

Caspase assay. Caspase3/7/8 activity was determined using Cell Meter ${ }^{\mathrm{TM}}$ Caspase 3/7 and Caspase 8 Activity Apoptosis Assay kits (AAT Bioquest, Inc.) according to the manufacturer's instructions (23). The results were read at $520 \mathrm{~nm}$ using a microplate reader (Bio-Rad Laboratories, Inc.) and expressed as fold change in caspase 3/7/8 activity from control.

Reverse transcription-quantitative PCR (RT-qPCR). Total RNA was extracted from cells (I/R model), using TRIzol (Invitrogen; Thermo Fisher Scientific, Inc.) and reverse transcribed to cDNA using Transcriptor First Strand cDNA Synthesis kit (Roche Molecular Systems, Inc.) according to manufacturer's protocol. qPCR was performed using the SYBR $^{\circledR}$ Green PCR Master Mix Reagent kit (Takara Bio, Inc.), and GAPDH was used for data normalization. The thermocycling conditions were as follows: $5 \mathrm{~min}$ at $95^{\circ} \mathrm{C}$, followed by $35-40$ cycles of $5 \mathrm{sec}$ at $95^{\circ} \mathrm{C}$ and $10 \mathrm{sec}$ at $60^{\circ} \mathrm{C}$. The fold-change of expression of the transcript mRNA was analyzed using the $2^{-\Delta \Delta \mathrm{Cq}}$ method (24). The primers used were as follows: CD36 forward, 5'-GAGCCATCTTTGAGCCTTCA-3' and reverse, 5'-TCAGATCCGAACACAGCGTA-3'; GADPH forward, 5'-GGAGCCAAAAGGGTCATCAT-3' and reverse, 5'-GTGATGGCATGGACTGTGGT-3'; autophagy-related 7 (ATG7) forward, 5'-CGGCTGAGATCTGGGACA-3' and reverse, 5'-AGCCAGATTGAGCGACTGAT-3' (all purchased from Invitrogen; Thermo Fisher Scientific, Inc.).

Western blot analysis. Hepatocytes (I/R model) were lysed with ice-cold Cell Lysis Buffer (cat. no. ab152163; Abcam) to obtain total protein, which was subsequently quantified using bicinchoninic acid protein assay. Protein samples $(20 \mu \mathrm{g} / \mathrm{lane})$ were then separated by $10 \%$ SDS-PAGE and transferred to polyvinylidene difluoride membranes (EMD Millipore; Merck KGaA). Following transfer, the membranes were blocked with $5 \%$ milk in Tris-buffered saline and incubated with the appropriate primary antibodies (all 1:1,000 dilution; LC3B, cat. no. 3868; ATG7, cat. no. 8558 and $\beta$-actin, cat. no. 4970; all from Cell Signaling Technology, Inc.) overnight at $4^{\circ} \mathrm{C}$. The membranes were subsequently incubated with horseradish peroxidase-conjugated secondary antibody (1:2,000 dilution; cat. no. 7074, Cell Signaling Technology, Inc.) and visualized using ECL Substrate Kit (Abcam). The stained protein bands were visualized using ChemiDoc XRS equipment (Bio-Rad Laboratories, Inc.), and quantified and analyzed using Quantity One software (version 4.5.2; Bio-Rad Laboratories, Inc.).

Small interfering (si)RNA transfection. Hepatocytes were resuspended $\left(1 \times 10^{6}\right.$ cells $\left./ \mathrm{ml}\right)$ in serum- and antibiotic-free siRNA Transfection Medium (cat. no. sc-36868; Santa Cruz Biotechnology, Inc.). CD36, ATG7 and control siRNAs (CD36, 5'-CTGTCCATCCCGCACCTGCG-3' and ATG7, 5'-CTC GCCGAGCTCGCCCA-3'; scramble control 5'-ACGTCT ATACGCCCA-3') were purchased from Invitrogen (Thermo 

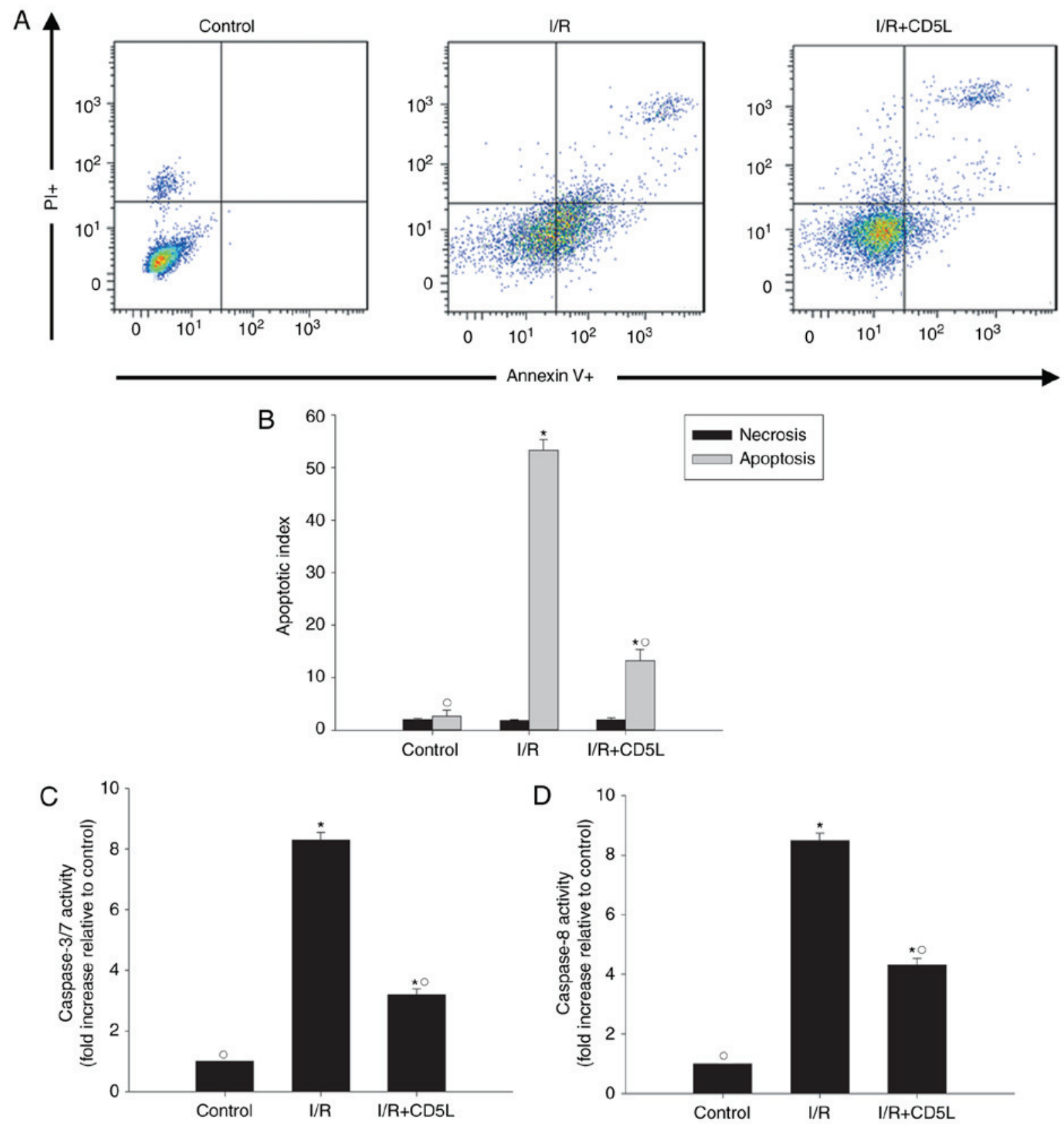

Annexin $\mathrm{V}_{+}$

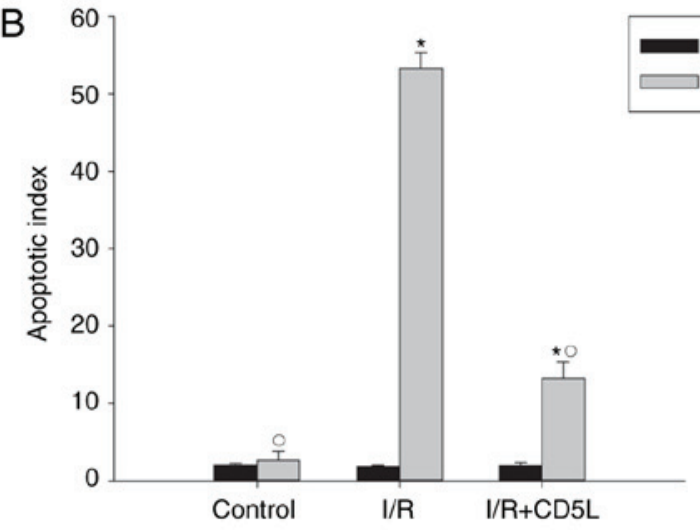

Necrosis

Apoptosis

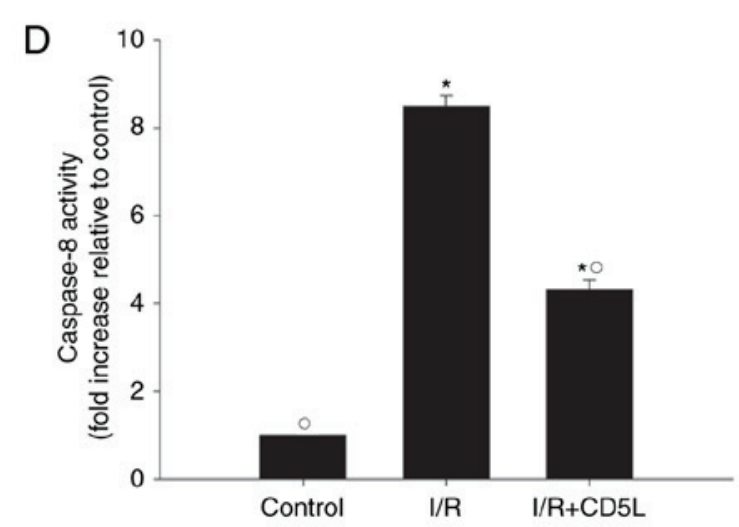

Figure 1. Recombinant CD5L inhibits I/R-induced hepatocyte apoptosis. (A) Representative images of Annexin V/PI flow cytometry analysis of apoptotic I/R injury model cells treated with CD5L. (B) Quantified flow cytometry results demonstrated that CD5L significantly reversed the apoptotic rate in I/R injury model cells. (C) Caspase 3/7 and (D) caspase 8 activity levels in hepatocyte cell lysates were measured using ELISA. Each column represents the mean \pm SD of three independent experiments. " $\mathrm{P}<0.05$ vs. control; ${ }^{\circ} \mathrm{P}<0.05$ vs. I/R. I/R, ischemia/reperfusion; CD5L, CD5-like; PI, propidium iodide.

Fisher Scientific, Inc.). Lipofectamine ${ }^{\circledR} 2000$ (Thermo Fisher Scientific, Inc.) was used to perform siRNA transfections $(100 \mathrm{nM})$ according to the manufacturer's protocol. Cells were analyzed at $48 \mathrm{~h}$ following transfection.

ROS production measurement. Intracellular production of ROS was detected by staining with 2',7'-dichlorofluorescin diacetate (DCFH-DA; Sigma-Aldrich; Merch KGaA). Following treatments, cells were washed thrice with sterile PBS and incubated with $10 \mu \mathrm{M}$ DCFH-DA for $30 \mathrm{~min}$ at $37^{\circ} \mathrm{C}$. The fluorescence intensity of the cells was measured using a fluorescence spectrophotometer at excitation and emission wavelengths of $488 \mathrm{~nm}$ and $525 \mathrm{~nm}$, respectively.
Superoxide dismutase (SOD) activity. SOD activity in treated hepatocytes was determined using a colorimetric Superoxide Dismutase Activity Assay Kit (cat. no. ab65354; Abcam) according to the manufacturer's protocol. Briefly, protein was isolated from hepatocytes using the lysis buffer as part of the kit, and SOD activity was measured in $10 \mu \mathrm{g}$ of total protein extract. Absorbance was measured at $450 \mathrm{~nm}$.

Glutathione peroxidase (GSH-Px) Assay. The level of GSH-Px was assayed using fluorometric green GSH/GSSG Ratio Detection Assay (cat. no. ab138881; Abcam) and performed as previously described (25). The absorbance of the samples was assessed using a spectrophotometer at $340 \mathrm{~nm}$. 

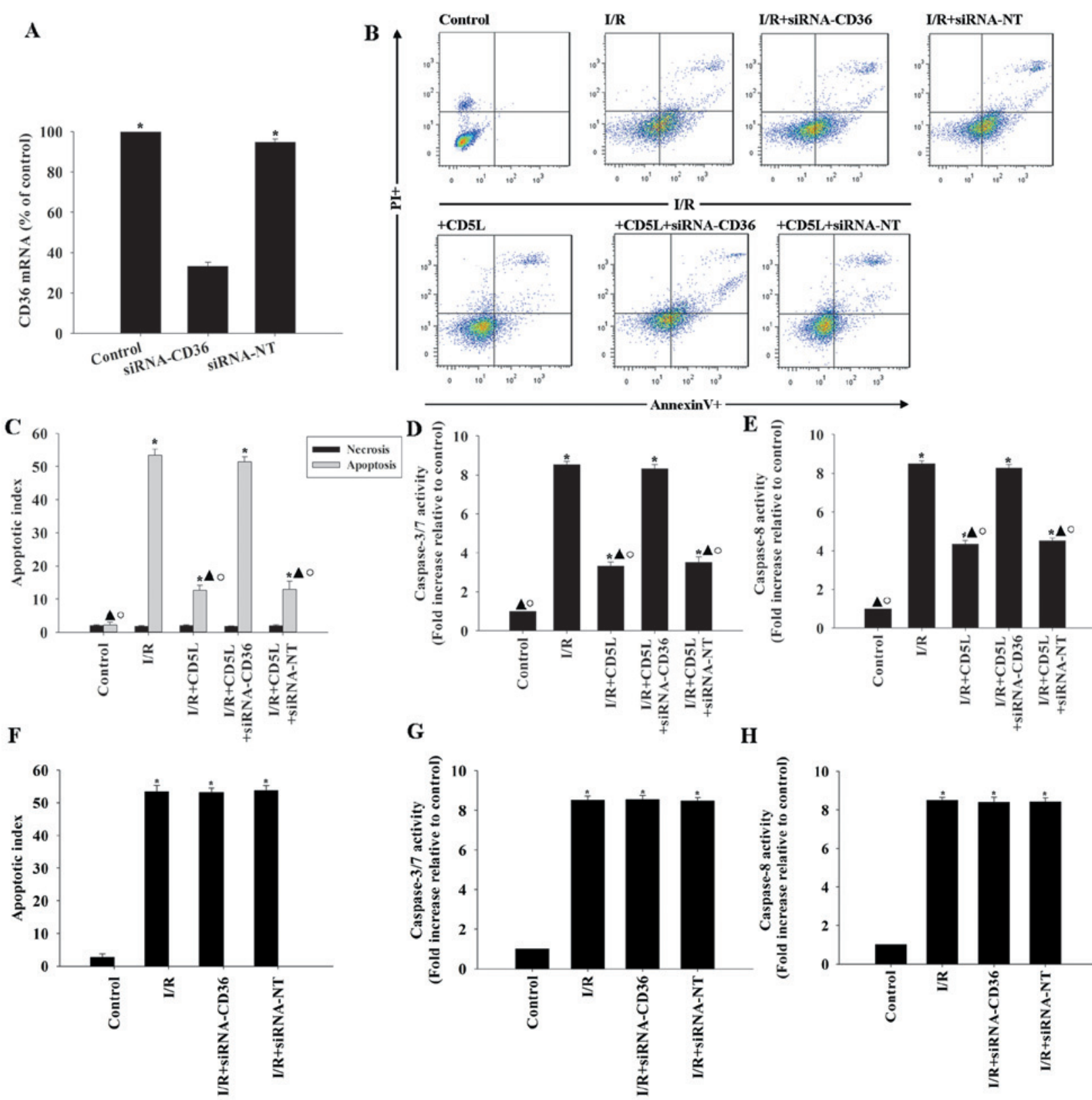

$\mathbf{H}$

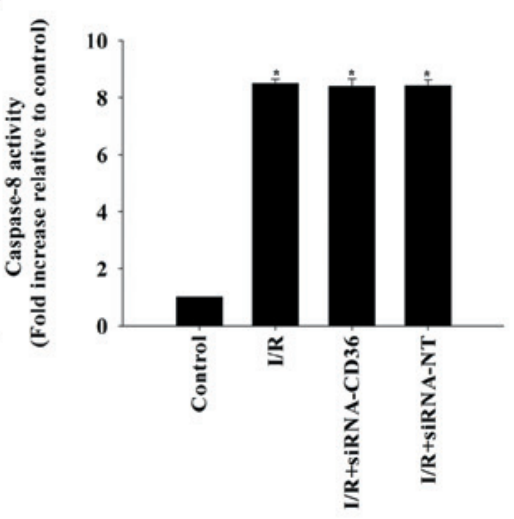

Figure 2. Inhibition of hepatocyte apoptosis by CD5L occurs via the CD36 receptor. (A-E) Hepatocytes were transfected with siRNA targeting CD36 or with siRNA-NT as a control. (A) Transfection efficiency was determined by reverse transcription-quantitative PCR. Each column represents the mean \pm SD of three independent experiments. "P<0.05 vs. siRNA-CD36. (B) Representative flow cytometric dot plots of apoptotic cells following Annexin V/propidium iodide staining and (C) quantification of apoptosis. (D) Caspase 3/7 and (E) caspase 8 activity levels in hepatocyte cell lysates were measured using ELISA. Each column represents the mean $\pm \mathrm{SD}$ of three independent experiments. ${ }^{*} \mathrm{P}<0.05$ vs. control; ${ }^{\wedge} \mathrm{P}<0.05$ vs. I/R; ${ }^{\circ} \mathrm{P}<0.05$ vs. I/R + siRNA-CD36. (F-H) Hepatocytes were transfected with siRNA against the CD36 transcript or with siRNA-NT as a control and incubated under I/R conditions. (F) Apoptosis was analyzed by flow cytometry. (G) Caspase 3/7 or $(\mathrm{H})$ caspase 8 activity from hepatocyte cell lysates were measured using ELISA. Each column represents the mean \pm SD of three independent experiments. ${ }^{*}<0.05$ vs. control. I/R, ischemia/reperfusion; CD5L, CD5-like; CD36, cluster of differentiation 36; siRNA, small interfering RNA; NT, non-targeting; PI, propidium iodide.

Catalase (CAT) activity assay. CAT activity in cells was determined using a colorimetric Catalase Activity Assay Kit (cat. no. ab83464; Abcam) according to the manufacturer's instructions. The absorbance of the samples was assessed using a spectrophotometer at $570 \mathrm{~nm}$.

Statistical analysis. Data are expressed as the mean \pm SD. SPSS version 19.0 (IBM Corp.) was used for statistical analysis. Differences among $\geq 3$ groups were tested by one-way analysis of variance and Tukey's test for multiple comparisons. Differences between two groups were evaluated by Student's t-test. $\mathrm{P}<0.05$ was considered to indicate a statistically significant difference.

\section{Results}

Recombinant CD5L inhibits I/R-induced hepatocyte apoptosis. As hepatocyte apoptosis has been implicated in I/R injury (26), the apoptotic rate of hepatocytes was tested in the I/R model in the present study. Annexin V-FITC fluorescence-activated cell sorting (FACS) results revealed that I/R-induced apoptosis compared with that in control cells, but this effect could be 
A
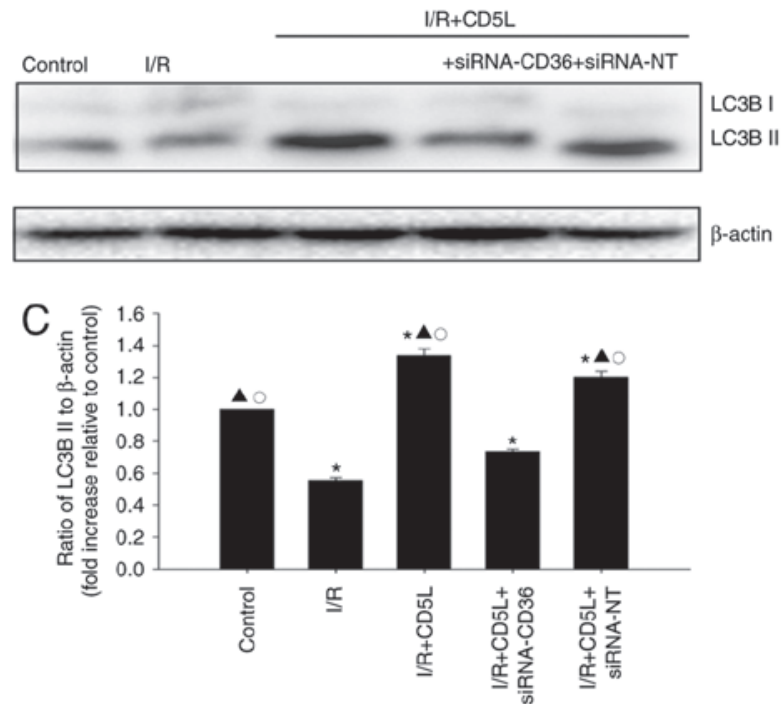

$\mathrm{E}$
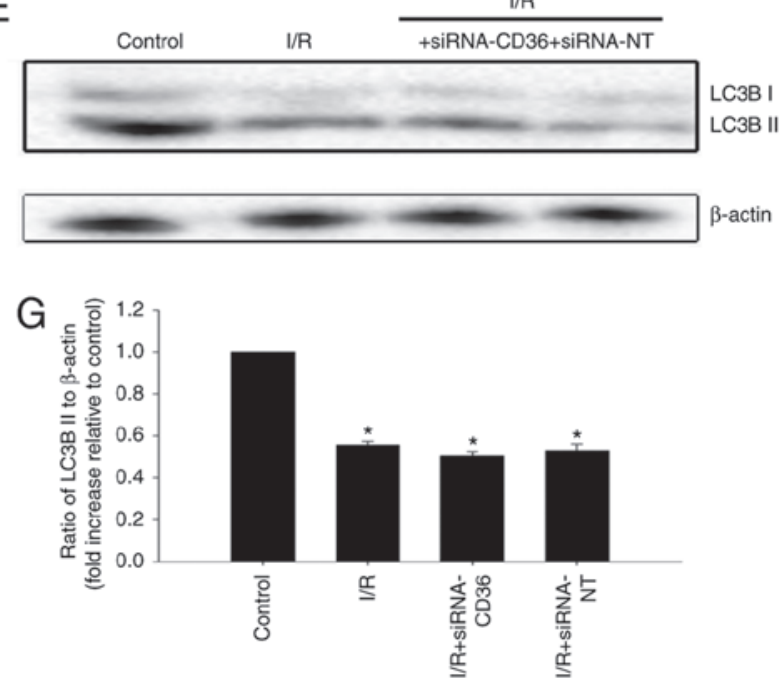

B

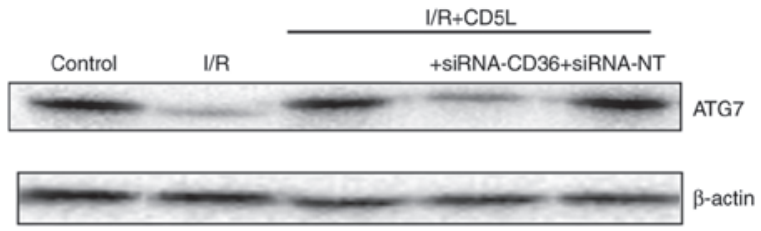

D

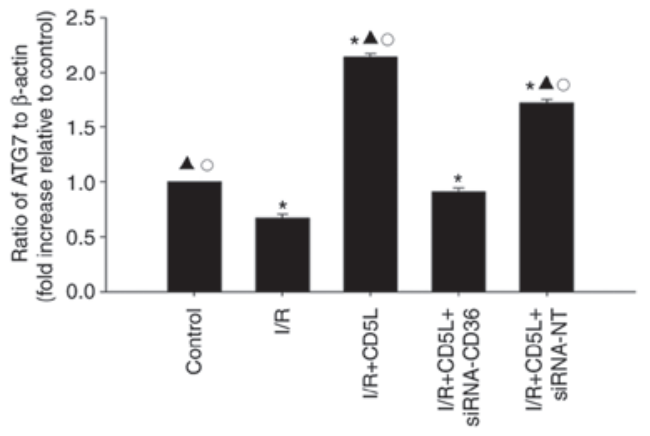

F
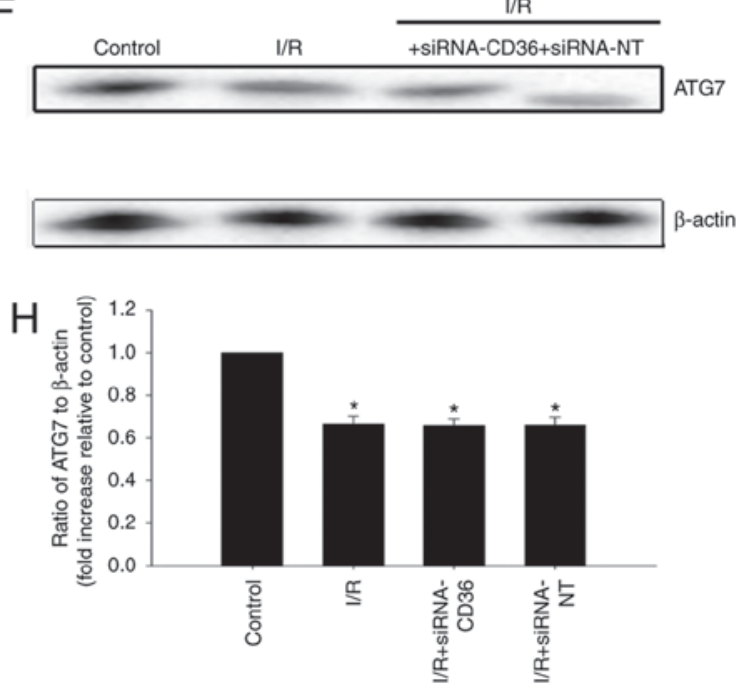

Figure 3. CD5L activates the cellular autophagy process. (A-D) Hepatocytes were transfected with siRNA-CD36 or siRNA-NT, incubated under I/R conditions and treated with CD5L. Cells without any treatment was used as control. (A and C) Representative western blots and quantification of the expression levels of LC3B-II and $\beta$-actin. (B and D) Representative western blots and quantification of the expression levels of ATG7 and $\beta$-actin. Each column represents the mean $\pm \mathrm{SD}$ of three independent experiments. ${ }^{*} \mathrm{P}<0.05$ vs. control; ${ }^{\mathrm{A}} \mathrm{P}<0.05$ vs. I/R; ${ }^{\circ} \mathrm{P}<0.05$ vs. I/R $+\mathrm{CD} 5 \mathrm{~L}+$ siRNA-CD36. (E-H) Hepatocytes were transfected with siRNA targeting CD36 or siRNA-NT as a control and incubated under I/R conditions. (E and G) Representative western blots and quantification of the expression levels of ATG7. (F and H) Representative western blots and quantification of the expression levels of $\beta$-actin. Each column represents the mean \pm SD of three independent experiments. * $\mathrm{P}<0.05$ vs. control. I/R, ischemia/reperfusion; CD5L, CD5-like; CD36, cluster of differentiation 36; siRNA, small interfering RNA; NT, non-targeting.

partially reversed by the application of recombinant CD5L (Fig. 1A and B). To further explore the antiapoptotic effects of CD5L, the changes in apoptosis marker caspases 3/7 and caspase 8 were measured following I/R induction with or without CD5L pretreatment. The results demonstrated that CD5L significantly reduced the caspase activity compared with untreated I/R model cells (Fig. 1C and D).

CD5L inhibits hepatocyte apoptosis via the CD36 receptor. $\mathrm{CD} 5 \mathrm{~L}$ activates the scavenger receptor $\mathrm{CD} 36$ on the cell surface to mediate the cellular protection process (16). Therefore, the role of CD36 in CD5L-induced hepatocyte protection was investigated. siRNAs targeting CD36 silenced its expression in hepatocytes by $>80 \%$ compared with the non-targeting siRNA (siRNA-NT) negative control (Fig. 2A). FACS analysis of CD36-silenced hepatocytes treated with CD5L compared with siRNA-NT-transfected cells treated with CD5L demonstrated an increase in apoptosis (Fig. 2B and C). In addition, silencing CD36 reversed the inhibition of caspase activity induced by CD5L (Fig. 2D and E). By contrast, siRNA-CD36 or siRNA-NT did not affect the apoptotic rates and caspase activity under I/R conditions (Fig. 2F-H).

CD5L has a cytoprotective effect by modulating autophagy. As the induction of autophagy is associated with an antiapoptotic effect during $\mathrm{I} / \mathrm{R}$ (15), the autophagic flux was examined in I/R hepatocytes by assessing the expression of LC3B-II, a marker for autophagy, by western blotting of total cell lysates. The present study also identified that ATG7 upregulation was also associated with protection against I/R liver injury. $\mathrm{I} / \mathrm{R}$ induction decreased LC3B-II and ATG7 levels in hepatocytes compared with the control group, whereas CD5L 
A

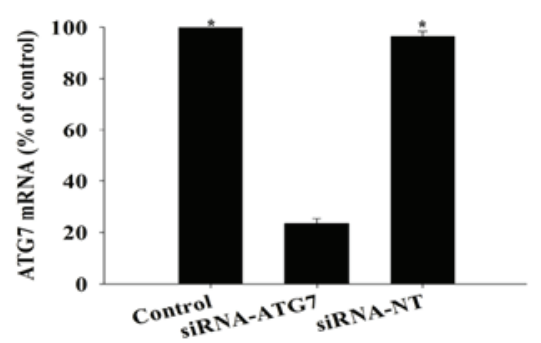

C

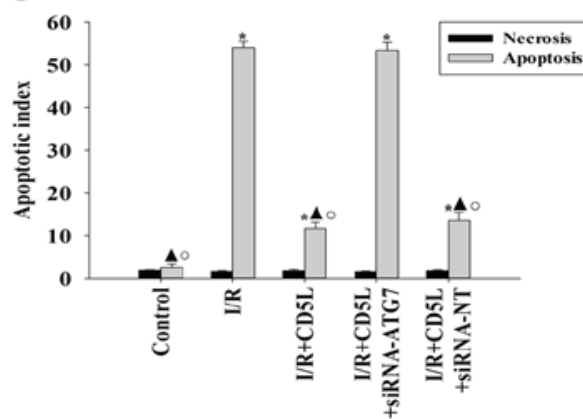

F

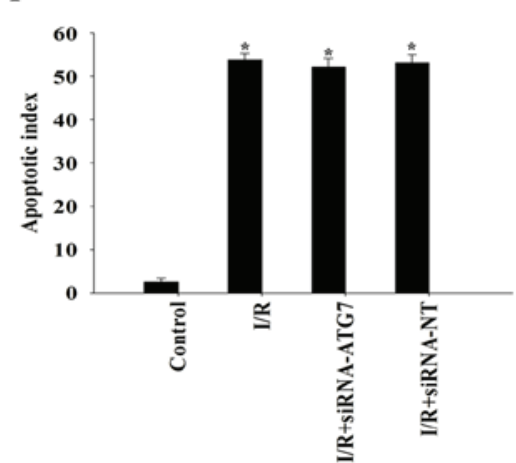

B
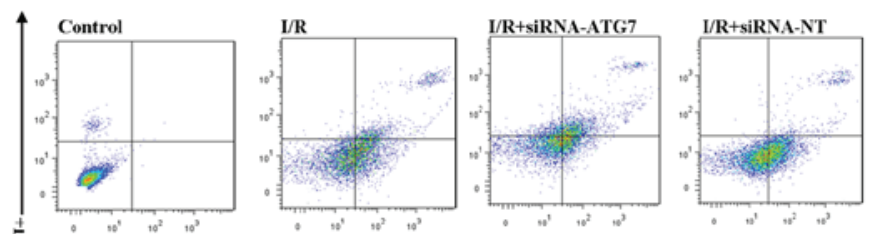

$\pm$

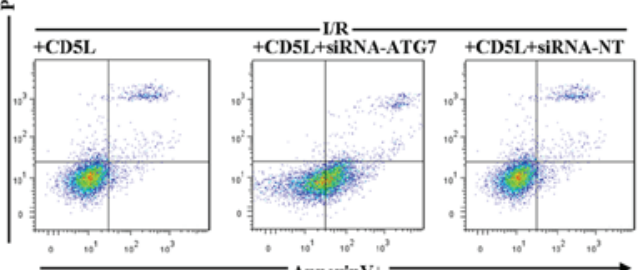

D
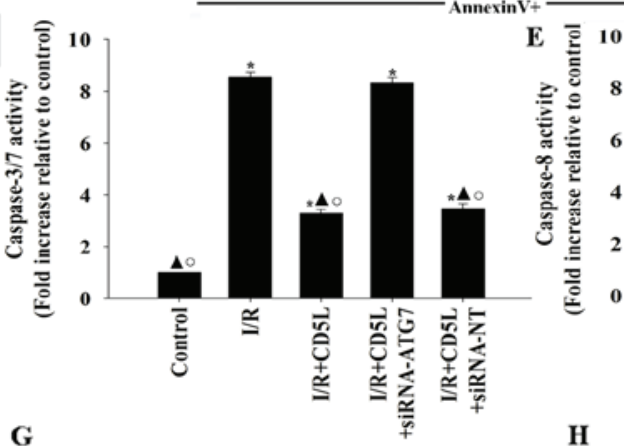
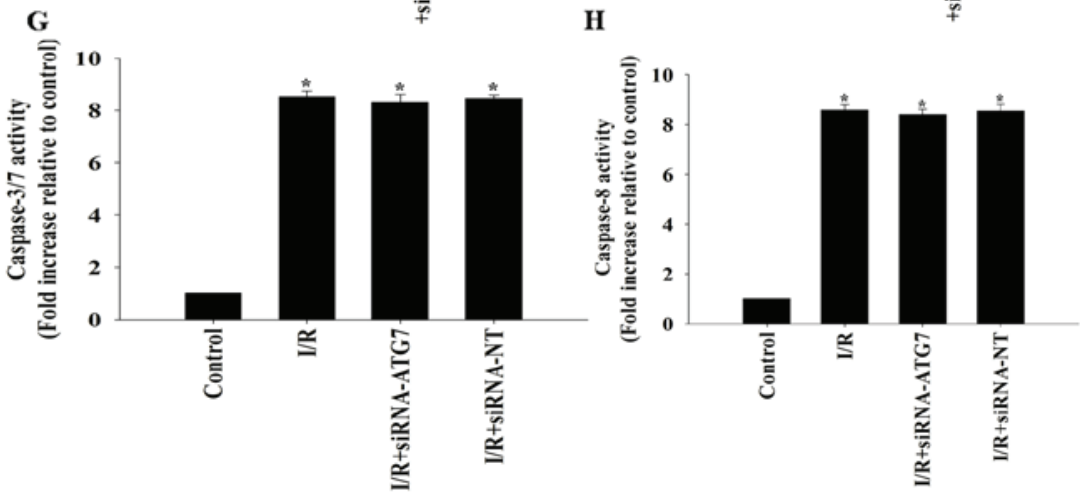

Figure 4. CD5L serves a cytoprotective function by modulating autophagy. (A-E) To determine the role of autophagy in the anti-apoptotic actions of CD5L, hepatocytes were transfected with siRNA against ATG7 or siRNA-NT. (A) The siRNA-mediated transfection efficiency was demonstrated by reverse transcription-quantitative PCR. Each column represents the mean \pm SD of three independent experiments. "P $<0.05$ vs. siRNA-ATG7. (B-E) Hepatocytes were incubated under I/R conditions and treated with CD5L. (B) Representative flow cytometric dot plots of apoptotic cells following Annexin V/propidium iodide staining and (C) quantification of apoptosis. (D) Caspase 3/7 and (E) caspase 8 activity levels in cell lysates were measured using ELISA. Each column represents the mean $\pm \mathrm{SD}$ of three independent experiments. ${ }^{*} \mathrm{P}<0.05$ vs. control; ${ }^{\wedge} \mathrm{P}<0.05$ vs. I/R; ${ }^{\circ} \mathrm{P}<0.05$ vs. I/R $+\mathrm{CD} 5 \mathrm{~L}+$ siRNA-ATG7. (F-H) Hepatocytes were transfected with siRNA targeting ATG7 or siRNA-NT as a control and incubated under I/R conditions. (F) Apoptosis was analyzed by flow cytometry. (G) Caspase 3/7 and (H) caspase 8 activity levels in hepatocyte cell lysates were measured using ELISA. Each column represents the mean \pm SD of three independent experiments. " $\mathrm{P}<0.05$ vs. control. I/R, ischemia/reperfusion; CD5L, CD5-like; ATG7, autophagy-related 7; siRNA, small interfering RNA; NT, non-targeting.

treatment increased LC3B-II (Fig. 3A and C) and ATG7 levels (Fig. 3B and D) compared with the I/R group; these results indicated that the induction of autophagy may occur upon CD5L activation. CD36 silencing reversed the expression of autophagy-related proteins induced by CD5L (Fig. 3E-H).

To confirm these results, experiments aimed at silencing the protein ATG7, an integral component of the autophagy process (27), were performed. siRNA transfection targeting ATG7 lowered the expression levels of ATG7 mRNA in hepatocytes (Fig. 4A). ATG7 silencing reversed the antiapoptotic effect of CD5L, and was associated with an increase in apoptosis compared with the control groups (Fig. 4B and C). In addition, following CD5L treatment, ATG7 silencing resulted in increased caspase activity compared with siRNA-NT-transfected cells (Fig. 4D and E). By contrast,
siRNA-ATG7 and siRNA-NT had no effects on apoptosis and caspase activity under I/R condition without CD5L treatment (Fig. 4F-H).

CD36 and ATG7 reverse the antioxidant effect of CD5L in hepatocytes. Since the oxidative stress is associated with I/R-related cellular damage (28), the effects of CD5L on ROS generation and antioxidant enzyme activity were examined. As demonstrated in Fig. 5A, I/R exposure significantly increased ROS generation in hepatocytes compared with that in the control group. In addition, antioxidant enzyme activity analysis revealed that the levels of SOD, GSH-Px and CAT in the $\mathrm{I} / \mathrm{R}$ groups were significantly reduced compared with those in the control groups (Fig. 5B-D). Following treatment with CD5L, ROS generation was 

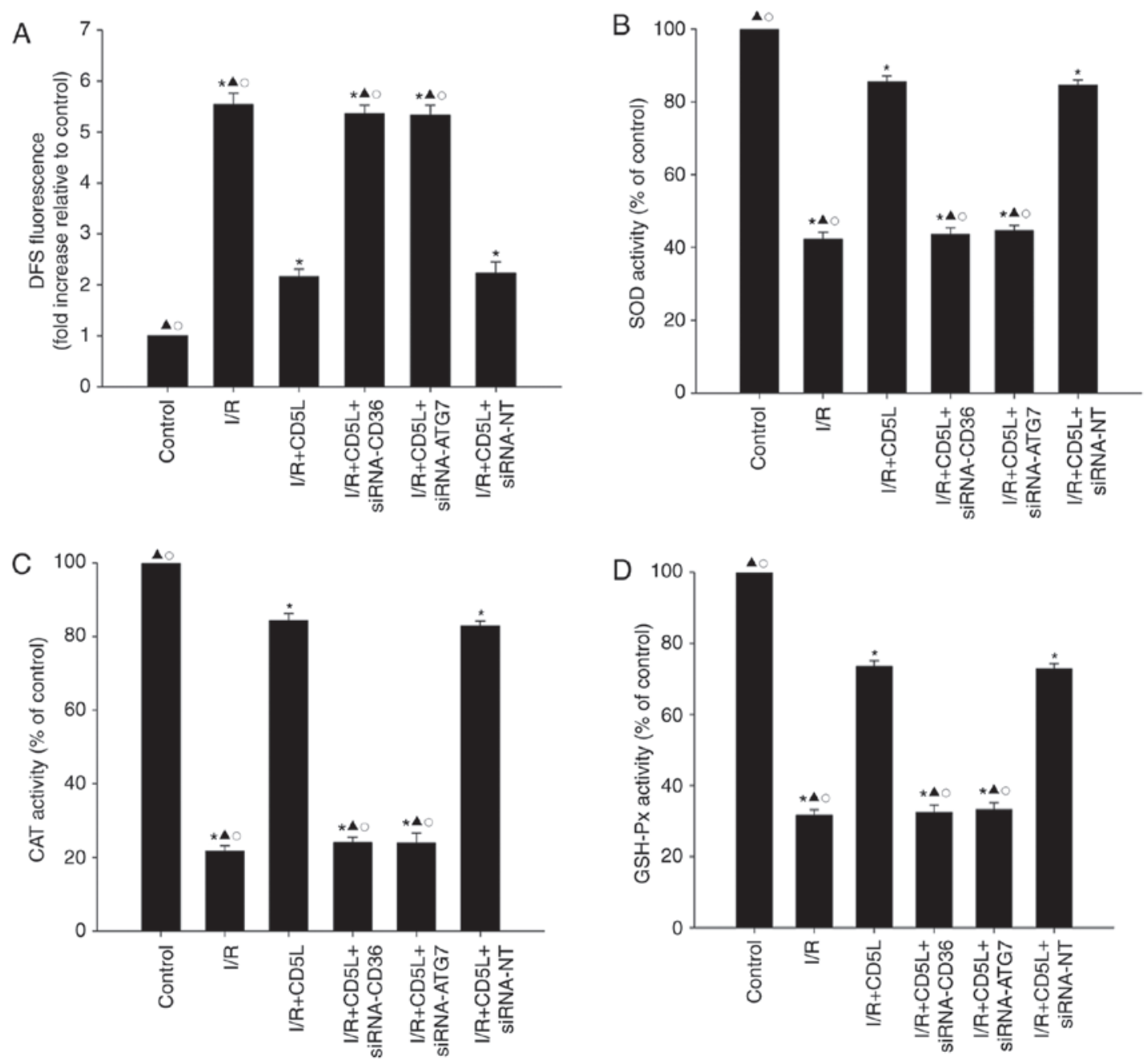

Figure 5. CD36 and ATG7 reverse the antioxidant effect of CD5L on hepatocytes. (A-D) Hepatocytes transfected with siRNA-CD36, siRNA-ATG7 or siRNA-NT and cultured under I/R conditions with or without CD5L. Hepatocytes without any treatment were used as controls. (A) Intracellular ROS production was analyzed by fluorescence spectrophotometry. Commercial kits were used to determine the levels of (B) SOD, (C) CAT and (D) GSH-Px activities in hepatocytes. Data represent the mean $\pm \mathrm{SD}$ from three independent experiments. ${ }^{*} \mathrm{P}<0.05$ vs. control; ${ }^{\Delta} \mathrm{P}<0.05$ vs. I/R $+\mathrm{CD} 5 \mathrm{~L} ;{ }^{\circ} \mathrm{P}<0.05$ vs. I/R $+\mathrm{CD} 5 \mathrm{~L}$ + siRNA-NT. DFS, dual fluorescent staining; I/R, ischemia/reperfusion; CD5L, CD5-like; CD36, cluster of differentiation 36; ATG7, autophagy-related 7; siRNA, small interfering RNA; NT, non-targeting; ROS, reactive oxygen species; SOD, superoxide dismutase; CAT, catalase; GHS-Px, glutathione peroxidase.

decreased, but antioxidant enzyme activity levels were increased compared with the I/R group; silencing either CD36 or ATG7 reversed the antioxidant effect of CD5L on hepatocytes (Fig. 5).

\section{Discussion}

Liver ischemic injury is of paramount importance in VIO applied during liver transaction (29). Liver hypoxia and ischemia affect liver cell homeostasis and function, which leads to metabolic alterations that may result in apoptosis (30). Ischemic stress-induced hepatocyte damage and cell death subsequently impair liver function (31). The present study demonstrated that hepatic injury induced cell damage and hepatocyte apoptosis.

CD5L is a $40-\mathrm{kDa}$ soluble glycoprotein that belongs to the scavenger receptor cysteine rich superfamily (32). CD5L is involved in a variety of biological processes, such as infection, atherosclerosis and apoptosis $(11,20)$. Various disease models, including cancer, have demonstrated that CD5L participates in cellular functions by preventing apoptosis $(33,34)$. Human CD5L has also been demonstrated to inhibit apoptosis in liver cancer cell lines in response to cisplatin by inducing autophagy (12). A previous study has suggested that CD5L serves cytoprotective effects by binding to the CD36 receptor (16). The results of the present study demonstrated that recombinant $\mathrm{CD} 5 \mathrm{~L}$ exhibited an antiapoptotic effect in I/R-induced apoptosis, and siRNA silencing of CD36 reversed this effect, which suggested that CD36 may be involved in the cytoprotective effects of CD5L.

Autophagy serves an important role in supporting hepato-cellular viability following I/R injury (21). Promotion of autophagy prevents mitochondrial dysfunction and cell death following reperfusion (35). A recent study has demonstrated that increasing the level of autophagy decreases hepatic I/R injury (36). Previous studies have reported that CD5L increases macrophage survival and liver cancer cell survival by enhancing autophagy $(12,16)$. These data indicate the participation of CD5L in promoting autophagy. In accordance with this, the results of the present study revealed that CD5L 
enhanced the expression of LC3B-II and ATG7 in hepatocytes. Additionally, silencing of ATG7, a key component of the autophagy signaling network, impaired the antiapoptotic effect of CD5L. These results support the hypothesis that CD5L promotes autophagy mechanisms in hepatocytes to induce an antiapoptotic effect.

Production of ROS, including superoxide, hydrogen peroxide and hydroxyl radicals, has been implicated in I/R injury (37). The results of the present study demonstrated that $\mathrm{I} / \mathrm{R}$ induced ROS generation and inhibited antioxidative enzyme activity. A previous study has suggested that CD5L induces anti-inflammatory effects by autophagy (20); therefore, the inhibitory effect of CD5L on oxidative stress was examined in the present study. In accordance with the result of previous study, CD5L inhibited the oxidative stress induced by I/R. In addition, autophagy is a major regulator of cell homeostasis and function through the modulation of oxidative stress under ischemic conditions (38); consistent with this, the present study revealed that when autophagic flux was blocked by ATG7 silencing in hepatocytes, the inhibition of oxidative stress by CD5L was partially reversed, which suggested that the CD5L-induced inhibition of oxidative stress may be autophagy-dependent.

In summary, the results of the present study demonstrated a protective effect of CD5L on I/R-induced hepatic injury through a CD36-dependent autophagic pathway, as well as inhibition of oxidative stress. These results indicate that CD5L may be a potential candidate for the treatment of $\mathrm{I} / \mathrm{R}$ injury.

\section{Acknowledgements}

Not applicable.

\section{Funding}

The current study was supported by The Natural Science Foundation of Zhejiang Province (grant no. LQ13H160022 to JL), the Science and Technology Program of Wenzhou Municipality (grant no. Y20140712 to JL) and the Science and Technology Program of Wenzhou Municipality (grant no. Y20190445 to LZ).

\section{Availability of data and materials}

All data generated or analyzed during this study are included in this published article.

\section{Authors' contributions}

JJL and WL made substantial contributions to the acquisition of data, analysis and interpretation of data. LZ was involved in conception and design of the study, and drafting the manuscript.

\section{Ethics approval and consent to participate}

The present study was approved by the Ethics Committee of Wenzhou Medical University, Wenzhou, China (approval no. WMU18825).

\section{Patient consent for publication}

Not applicable.

\section{Competing interests}

The authors declare that they have no competing interests.

\section{References}

1. Starlinger P, Assinger A, Haegele S, Wanek D, Zikeli S, Schauer D, Birner P, Fleischmann E, Gruenberger B, Brostjan C and Gruenberger T: Evidence for serotonin as a relevant inducer of liver regeneration after liver resection in humans. Hepatology 60: 257-266, 2014.

2. Schiergens TS, Stielow C, Schreiber S, Hornuss C, Jauch KW, Rentsch $\mathrm{M}$ and Thasler WE: Liver resection in the elderly: Significance of comorbidities and blood loss. J Gastrointest Surg 18: 1161-1170, 2014.

3. Duval H, Mbatchi SF, Grandadam S, Legendre C, Loyer P, Ribault C, Piquet-Pellorce C, Guguen-Guillouzo C, Boudjema K and Corlu A: Reperfusion stress induced during intermittent selective clamping accelerates rat liver regeneration through JNK pathway. J Hepatol 52: 560-569, 2010

4. Olthof PB, Reiniers MJ, Dirkes MC, Gulik TMV and Golen RFV: Protective mechanisms of hypothermia in liver surgery and transplantation. Mol Med 21: 833-846, 2016.

5. Castellaneta A, Yoshida O, Kimura S, Yokota S, Geller DA, Murase N and Thomson AW: Plasmacytoid dendritic cell-derived IFN- $\alpha$ promotes murine liver ischemia/reperfusion injury by induction of hepatocyte IRF-1. Hepatology 60: 267-277, 2014.

6. Zuo S, Kong D, Wang C, Liu J, Wang Y, Wan Q, Yan S, Zhang J, Tang J, Zhang Q, et al: CRTH2 promotes endoplasmic reticulum stress-induced cardiomyocyte apoptosis through m-calpain. EMBO Mol Med 10: e8237, 2018.

7. Sun P, Zhang P, Wang PX, Zhu LH, Du Y, Tian S, Zhu X and Li H: Mindin deficiency protects the liver against ischemia/reperfusion injury. J Hepatol 63: 1198-1211, 2015.

8. Rao J, Qian X, Li G, Pan X, Zhang C, Zhang F, Zhai Y, Wang X and Lu L: ATF3-mediated NRF2/HO-1 signaling regulates TLR4 innate immune responses in mouse liver ischemia/reperfusion injury. Am J Transplant 15: 76-87, 2015.

9. Zhang Y, Liu X, She ZG, Jiang DS, Wan N, Xia H, Zhu XH, Wei X, Zhang XD and Li H: Interferon regulatory factor 9 is an essential mediator of heart dysfunction and cell death following myocardial ischemia/reperfusion injury. Basic Res Cardiol 109: 434, 2014.

10. Ozawa T, Maehara N, Kai T, Arai S and Miyazaki T: Dietary fructose-induced hepatocellular carcinoma development manifested in mice lacking apoptosis inhibitor of macrophage (AIM). Genes Cells 21: 1320-1332, 2016

11. Sanjurjo L, Aran G, Roher N, Valledor AF and Sarrias MR: AIM/CD5L: A key protein in the control of immune homeostasis and inflammatory disease. J Leukoc Biol 98: 173-184, 2015.

12. Aran G, Sanjurjo L, Bárcena C, Simon-Coma M, Téllez É, Vázquez-Vitali M, Garrido M, Guerra L, DíazE, Ojanguren I, et al: CD5L is upregulated in hepatocellular carcinoma and promotes liver cancer cell proliferation and antiapoptotic responses by binding to HSPA5 (GRP78). FASEB J 32: 3878-3891, 2018.

13. Wang C, Yosef N, Gaublomme J, Wu C, Lee Y, Clish CB, Kaminski J, Xiao S, Meyer Zu Horste G, Pawlak M, et al: CD5L/AIM regulates lipid biosynthesis and restrains Th17 cell pathogenicity. Cell 163: 1413-1427, 2015.

14. Xiao J, Ke ZP, Shi Y, Zeng Q and Cao Z: The cardioprotective effect of thymoquinone on ischemia-reperfusion injury in isolated rat heart via regulation of apoptosis and autophagy. J Cell Biochem 119: 7212-7217, 2018.

15. Li X, Huang Q, Wang $M$, Yan X, Song X, Ma R, Jiang R, Zhao D and Sun L: Compound K inhibits autophagy-mediated apoptosis through activation of the PI3K-Akt signaling pathway thus protecting against ischemia/reperfusion injury. Cell Physiol Biochem 47: 2589-2601, 2018.

16. Sanjurjo L, Amézaga N, Aran G, Naranjo-Gómez M, Arias L, Armengol C, Borràs FE and Sarrias MR: The human CD5L/AIM-CD36 axis: A novel autophagy inducer in macrophages that modulates inflammatory responses. Autophagy 11: 487-502, 2015. 
17. van Golen RF, van Gulik TM and Heger M: Mechanistic overview of reactive species-induced degradation of the endothelial glycocalyx during hepatic ischemia/reperfusion injury. Free Radic Biol Med 52: 1382-1402, 2012.

18. van Golen RF, van Gulik TM and Heger M: The sterile immune response during hepatic ischemia/reperfusion. Cytokine Growth Factor Rev 23: 69-84, 2012.

19. de Graaf W, Heger M, Spruijt O, Maas A, de Bruin K, Hoekstra R, Bennink RJ and van Gulik TM: Quantitative assessment of liver function after ischemia-reperfusion injury and partial hepatectomy in rats. J Surg Res 172: 85-94, 2012.

20. Sanjurjo L, Aran G, Téllez É, Amézaga N, Armengol C, López D, Prats C and Sarrias MR: CD5L promotes M2 macrophage polarization through autophagy-mediated upregulation of ID3. Front Immunol 9: 480, 2018.

21. Biel TG, Lee S, Flores-Toro JA, Dean JW, Go KL, Lee MH, Law BK, Law ME, Dunn WA Jr, Zendejas I, et al: Sirtuin 1 suppresses mitochondrial dysfunction of ischemic mouse livers in a mitofusin 2-dependent manner. Cell Death Differ 23: 279-290, 2016

22. Yuan J, Chen M, Xu Q, Liang J, Chen R, Xiao Y, Fang M and Chen L: Effect of the diabetic environment on the expression of MiRNAs in endothelial cells: Mir-149-5p restoration ameliorates the high glucose-induced expression of TNF- $\alpha$ and ER stress markers. Cell Physiol Biochem 43: 120-135, 2017.

23. Liu Y, Xiong Y, Xing F, Gao H, Wang X, He L, Ren C, Liu L, So KF and Xiao J: Precise regulation of miR-210 is critical for the cellular homeostasis maintenance and transplantation efficacy enhancement of mesenchymal stem cells in acute liver failure therapy. Cell Transplant 26: 805-820, 2017.

24. Livak KJ and Schmittgen TD: Analysis of relative gene expression data using real-time quantitative PCR and the 2(-Delta Delta C(T)) method. Methods 25: 402-408, 2001.

25. Szabó K, Gesztelyi R, Lampé N, Kiss R, Remenyik J Pesti-Asbóth G, Priksz D, Szilvássy Z and Juhász B: Fenugreek (trigonella foenum-graecum) seed flour and diosgenin preserve endothelium-dependent arterial relaxation in a rat model of early-stage metabolic syndrome. Int J Mol Sci 19: E798, 2018.

26. Guo WZ, Fang HB, Cao SL, Chen SY, Li J, Shi JH, Tang HW, Zhang Y, Wen PH, Zhang JK, et al: Steap3 deficiency in hepatocytes protects the liver against ischemia/reperfusion injury by suppressing TAK1. Hepatology: Aug 8,2019 (Epub ahead of print).

27. Geng J and Klionsky DJ: The Atg8 and Atg12 ubiquitin-like conjugation systems in macroautophagy 'Protein modifications: Beyond the usual suspects' review series. EMBO Rep 9: 859-864, 2008.
28. $\mathrm{Mu} \mathrm{H}$ and Wang Y: Collagen peptide modified carboxymethyl cellulose as both antioxidant drug and carrier for drug delivery against retinal ischaemia/reperfusion injury. J Cell Mol Med 22: 5008-5019, 2018.

29. Abt P, Crawford M, Desai N, Markmann J, Olthoff K and Shaked A: Liver transplantation from controlled non-heart-beating donors: An increased incidence of biliary complications. Transplantation 75: 1659-1663, 2003.

30. Jaeschke $\mathrm{H}$ and Lemasters JJ: Apoptosis versus oncotic necrosis in hepatic ischemia/reperfusion injury. Gastroenterology 125: 1246-1257, 2003

31. Bilzer $M$ and Gerbes AL: Preservation injury of the liver: Mechanisms and novel therapeutic strategies. J Hepatol 32: 508-515, 2000.

32. Gebe JA, Kiener PA, Ring HZ, Li X, Francke U and Aruffo A: Molecular cloning, mapping to human chromosome 1 q21-q23, and cell binding characteristics of Spalpha, a new member of the scavenger receptor cysteine-rich (SRCR) family of proteins. J Biol Chem 272: 6151-6158, 1997.

33. Li Y, Qu P, Wu L, Li B, Du H and Yan C: Api6/AIM/Spa/CD5L overexpression in alveolar type II epithelial cells induces spontaneous lung adenocarcinoma. Cancer Res 71: 5488-5499, 2011.

34. Zou T, Garifulin O, Berland R and Boyartchuk VL: Listeria monocytogenes infection induces prosurvival metabolic signaling in macrophages. Infect Immun 79: 1526-1535, 2011.

35. Chun SK, Lee S, Flores-Toro J, U RY, Yang MJ, Go KL, Biel TG, Miney CE, Pierre Louis S, Law BK, et al: Loss of sirtuin 1 and mitofusin 2 contributes to enhanced ischemia/reperfusion injury in aged livers. Aging Cell 17: e12761, 2018.

36. Tan R, Tian H, Yang B, Zhang B, Dai C, Han Z, Wang M, Li Y, Wei L, Chen D, et al: Autophagy and Akt in the protective effect of erythropoietin helix B surface peptide against hepatic ischaemia/reperfusion injury in mice. Sci Rep 8: 14703, 2018.

37. Jaeschke H: Molecular mechanisms of hepatic ischemia-reperfusion injury and preconditioning. Am J Physiol Gastrointest Liver Physiol 284: G15-G26, 2003.

38. Nishida K, Kyoi S, Yamaguchi O, Sadoshima J and Otsu K: The role of autophagy in the heart. Cell Death Differ 16: 31-38, 2009.

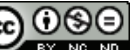

This work is licensed under a Creative Commons Attribution-NonCommercial-NoDerivatives 4.0 International (CC BY-NC-ND 4.0) License. 